\section{EMBRYRIDDLE \\ Aeronautical University}

SCHOLARLY COMMONS
International Journal of Aviation, Aeronautics, and Aerospace

Volume 1 | Issue 1

Article 1

$1-27-2014$

\title{
SMS - Reaching beyond low hanging fruit
}

Stewart Schreckengast

University of South Australia, stewart.schreckengast@unisa.edu.au

Follow this and additional works at: https://commons.erau.edu/ijaaa

Part of the Educational Methods Commons, and the Transportation Law Commons

\section{Scholarly Commons Citation}

Schreckengast, S. (2014). SMS - Reaching beyond low hanging fruit. International Journal of Aviation, Aeronautics, and Aerospace, 1(1). https://doi.org/10.15394/ijaaa.2014.1002

This Article is brought to you for free and open access by the Journals at Scholarly Commons. It has been accepted for inclusion in International Journal of Aviation, Aeronautics, and Aerospace by an authorized administrator of Scholarly Commons. For more information, please contact commons@erau.edu. 


\section{SMS - Reaching beyond low hanging fruit}

\section{Cover Page Footnote}

I would like to acknowledge my initial accident investigation training through the Naval Safety School along with Safety Management Systems training through the Federal Aviation Administration and the International Civil Aviation Organization. The wealth of experience and untiring efforts to standardize and harmonize International best practices is essential to the success of further safety development. 
The International Civil Aviation Organization (ICAO) has established aviation accident and incident investigation obligations within Article 26 of its Convention on International Civil Aviation. This is further developed in ICAO Annex 13 - Aircraft Incident and Incident Investigation and Annex 19 - Safety Management. The ICAO member States have implemented these international aviation investigation standards in various regulations, policies and procedures that have traditionally focused on the most severe events. Further implementation of aviation safety investigations into less severe events has significant opportunities to enhance aviation safety and operational efficiency.

\section{Definitions}

- Incident. An event, other than an accident, associated with the operation of an aircraft which affects or could affect the safety of operation.

- Investigation. A process conducted for the purpose of accident or incident prevention which includes the gathering and analysis of information, the drawing of conclusions, the determination of causes and/or contributing factors and, when appropriate, the making of safety recommendations.

- Occurrence. An adverse event or condition, other than an accident or incident, associated with an aircraft which affects or could affect operational safety.

- Safety Recommendation. A proposal of a safety investigation based on information derived from an investigation, made with the intention of preventing accidents and incidents and which in no case creates a presumption of blame or liability for an accident or incident. In addition to safety recommendations arising from an investigation, safety recommendations may result from diverse sources, including safety studies. These recommendations may further be used to identify system vulnerability, develop strategies for change, and prioritize the investment of safety resources (ICAO, 2013b).

\section{Service Provider Organizations}

In general, revenue producing aviation organizations, service providers, have an obligation to establish a safety program that includes a proactive element to identify and resolve deficiencies. The State Safety Program (SSP) and Civil Aviation Authority (CAA) should establish regulations for a Safety Management System (SMS) in approved training organizations, commercial air transport, maintenance organizations, air traffic services and aerodromes (ICAO, 2013b). This should also extend to international general aviation operators of large or turbojet aircraft. Included in this obligation is a foundation to monitor and 
respond to adverse events such as accidents and incidents utilizing traditional safety investigation procedures. These safety investigations may be covered by separate regulatory material from the transportation safety investigation authority.

It is important to note that many States provide separate safety and enforcement investigations with separate utilization of the outcomes from such programs. The policies and procedures of safety investigations should include protections or prohibitions from the use of such material for enforcement actions under ICAO Annex 19 Attachment B. The policies of the regulator can be used for administrative or enforcement actions.

When incidents do not meet the reporting obligations under safety or enforcement policies, the service provider should conduct an internal investigation to identify safety deficiencies and implement safety mitigation in order to ensure continued safety improvements and thus support safer and more efficient operations. Guidance on the implementation of SMS and safety investigations is also provided in FAA Advisory Circulars 120-92A for air carriers. Such investigations may vary in complexity but the documentation of these safety reviews is essential to build the foundation for future trend analysis and to meet the organization's quality assurance and SMS obligations. These investigative techniques also reinforce the organization's safety reporting culture and provide training experience for the safety investigators.

The reinforcement of the safety culture also helps institutionalize the limits of acceptable and unacceptable behavior (CASA, 2009a). The Counselling/discipline decision chart in Appendix B of the CASA SMS Toolkit Booklet 2 provides a systematic matrix of how incidents and occurrences might be resolved within the organization and assist in the identification of why such adverse events happened and might be resolved. Workers and management thus seek to identify unsafe or inefficient activities and resolve these deficiencies. The safety risks are solicited through an active safety reporting system and documented in a Safety Risk Registry. Management encourages such reporting and understands that it is better to identify an unsafe condition than ignore it. Everyone understands the necessity to comply with established safety obligations, continually update those safety obligations and ensure that employees respect such obligations. Those who decide not to comply with safety obligations and commit a willful violation should expect to receive both disrespect from their fellow workers and administrative actions from management. 


\section{Aviation Organization Policy Statement}

The organization should provide a safety policy that ensures the following:

- Conduct objective and independent investigations of incidents by documenting the facts, conditions, and circumstances of such occurrences without implying blame or liability;

- Identify safety deficiencies during investigations with the view toward prevention of future occurrences;

- As appropriate, disseminate the findings of the investigation and implement actions to eliminate or reduce risks posed by identified safety deficiencies.

Aviation service providers are part of an extraordinarily safe industry. As such, the flying public and regulators have placed greater safety obligations upon their operations. As discussed by Gessell and Dempsey (2011) non-aviation activities generally operate under a condition known as Ordinary Care which has been defined in the court cases Brown v Kendal and Osborne v Montgomery as "that kind and degree of care, which prudent and cautious men would use, such as is required by the exigency of the case, and such as is necessary to guard against probable dander." However, aviation service providers generally must provide and Extraordinary Duty of Care that holds them to a highest duty to their passengers, higher than that of reasonable care, by the court cases of O'Leary $v$ American Airlines and Krasnow v National Airlines.

General aviation also has greater than ordinary care requirements. These are often enforced using such regulations as the Federal Aviation Regulation FAR $\$ 1.13$ - Careless or reckless operation: No person may operate an aircraft in a careless or reckless manner so as to endanger the life or property of another.

\section{Legislative Requirements}

\section{ICAO Standards and Recommended Practices (SARPs)}

ICAO member States are obliged to conduct aircraft accident and serious incident investigation to comply with Article 26 of the Convention on International Civil Aviation. This obligation is enacted by State laws and regulations. Such legislation must establish an accident investigation authority and the processes identified in ICAO Annex 13 - Aircraft Accident and Incident Investigation. Furthermore, the newly published Annex 19 extends these SARPs to include the obligation for aviation service providers such as aviation training 
organizations and airlines to implement investigations into less severe deficiencies identified through their SMS and safety data collection applications.

Early aviation accident investigation evolved from maritime accident investigation. Under the Rules of the Sea, the ship's captain was ultimately responsible for the safety of the ship. Similarly, aviation accident investigation placed the responsibility of the aircraft in the captain's hands unless some extensive mechanical failure could be identified whereby the manufacturer shared the responsibility. Through the advances in manufacturing and regulatory oversight, this scheme of human error has remained the major causation of aviation accidents and incidents. Many investigative advances have been promoted with greater emphasis on non-reportable incidents and occurrences in order to mitigate safety risk to an acceptable level and to share the obligation of continual safety improvement throughout the aviation industry.

The development of the concept of accident causation has also aided in the understanding of human and organizational errors and the methodology for overcoming these deficiencies. Such deficiencies can range from active failures, either through actions or inactions, to unidentified or latent conditions that are often dormant until some triggering event is introduced, such as an autopilot failure to maintain a safe flight envelope when icing is encountered (Reason, 1990). Under the concept of capturing the low hanging fruit, many incident or occurrence investigations might settle for introducing additional training or internal policies to overcome a systemic deficiency. However, if these were more fully investigated using the methodology of an accident investigation, the recommendations might be more beneficial by changing technology or operating limitations of the aircraft. Again as example, an autopilot that might not be able to function in icing conditions might have a procedure to disconnect the autopilot and manually fly the aircraft, or limit the duration in which the aircraft could operate in icing conditions. In reality, either the technology of the aircraft to operate in icing conditions or the autopilot functions should be modified to mitigate the risk of adverse flight performance in icing conditions. Thus the obligation for the pilot to exercise exceptional flying skills would not be a necessary recommendation. This also reduces the need for the organization to develop special techniques to overcome deficiencies and thus accept such higher risks - a process known as normalization of deviance. This might be successful for a period of time when highly experienced pilots use their superior skills to overcome such marginal operations; however, over time, new personnel will attempt to emulate these normalized deviances with potentially adverse outcomes. 


\section{Investigation Objective and Independence}

ICAO Annex 13 establishes that accident and incident investigations must be objective, independent of outside influence and must also be so accepted by the service provider organization. ICAO Annex 13, Chapter 3, paragraph 3.1 - states that the sole objective of an incident investigation shall be the prevention of similar incidents. It is not the purpose of apportioning blame or liability. Chapter 5, paragraph 5.4 - further states that incident investigation authority shall have independence in the conduct of the investigation and have unrestricted authority over it; and, shall be separate from enforcement or administrative actions to apportion blame or liability. Insulation from administrative action should also be afforded to service provider internal safety investigations when such studies are below the reporting obligations of the State (AAIB, 2013). The US National Transportation Safety Board (NTSB) Part 830 also ensures that their Accident Investigation Reports can only be used for safety; however, factual information such as air traffic radar recordings may also be shared with the regulator, the Federal Aviation Administration (FAA), for both safety and enforcement actions. The report and investigation obligations for air carriers, the FAA and NTSB are further discussed in FAA Advisory Circular AC 120-30A.

\section{Investigation Policy and Procedures}

The service provider's policy should include the obligation to support an investigation by the regulator of all reportable accidents and incidents and to internally investigate the circumstances of other incidents not meeting that severity. If during an internal safety investigation or study of safety deficiencies the service provider identifies conditions that may impact external organizations, it should take immediate actions to disseminate such safety information.

For every accident or serious incident, there are perhaps hundreds of minor occurrences that have the potential to become accidents. The evaluation of hazard reports must ensure that the investigation is compliant with the safety policy of the organization to achieve an acceptable level of safety. Such hazards are captured in the Risk Registry for prioritization. An example of this process is discussed in the CASA SMS Toolkit Booklet 3. Since resources to conduct such investigations are often limited, there should be a proportional expenditure of resources with the estimated level of safety risk. Regardless of the potential for mitigation of a safety deficiency, a minimum of data should be collected in order to establish a foundation for future trend analysis. 
The service provider's investigators should have unhampered access to incident information or aircraft components. The investigators should also solicit support from equipment manufacturers, government agencies and experts as needed. The development of positive relations during normal operations will facilitate such support during adverse events. This will include permission to photograph and document components and take statements to preclude loss of vital information. Such data collection must also respect the privacy of individual medical records or other protected or limited access material.

Statements from witnesses and those involved in an incident should be insulated from enforcement through voluntary reporting systems such as the National Aeronautics and Space Administration (NASA) Aviation Safety Reporting Program which provides a waiver of enforcement by FAR $\$ 91.25$ except where the information relates to an aircraft accident or intentional violation. Similar insulation is provided in the Australian ATSB Act 2003 and European Union Article 19 of the Investigation Regulation No 996/2010 Directive 94/56/EC. An international collaboration on SMS has been established by the European Aviation Safety Agency (EASA), the FAA Office of Aviation Safety (AVS), ICAO, and Transport Canada Civil Aviation (TCCA). In 2009 these organizations established the Safety Management International Collaboration Group (SM ICG) to support the implementation and harmonization of SMS.

\section{Investigation Coordination}

Aviation service providers should actively conduct studies or safety surveys in order to provide adequate training, to identify deficiencies and to build confidence in the safety review process. These reviews also build a foundation for development of plans of action for more severe incident or accident. The SMS committee and safety action group may also require supplemental support from government and industry organizations. Thus consideration must be provided for protection of individuals reporting deficiencies and limitations to incident information source disclosure (ICAO, 2013a).

The organization's safety investigators should be issued policy guidance as to their authority and responsibility. The investigators should also be furnished with guidance material that can be provided to witnesses and participants in the investigation in order to protect the disclosure of the material gathered. Similarly, the use of outside experts can be established through letters of understanding or contracts which should caution them of their obligation to protect investigation material from disclosure. 


\section{Investigator Training}

The service provider should have appropriate investigator training. Such training should include an initial overview in investigation management and witness interviewing, followed by on-the-job training under an experienced investigator (ICAO, 2003). This can then be augmented by a formal incident investigation course and more advanced training in specialties such as flight data analysis and human factors (CASA, 2009b).

\section{Investigator Equipment}

In addition to individual training, investigators must be provided with certain equipment and have policies and agreements in place for access to this equipment such as flight data analysis and flight reconstruction applications. They should also receive training and equipment for incident site work in support of more severe incidents or accidents away from the service provider's normal location of operations. The ICAO Manual of Aircraft Accident and Incident Investigation, Part I (Doc 9756), has information related to an investigation field kit and protective equipment for potential hazards at an incident site.

It is essential to consider all aspects of a prior incident before attempting to re-enact an incident for study. Unfortunately many accidents or incidents have been repeated during the investigation of prior events.

\section{Incident Prevention Measures}

ICAO Annex 13, Chapter 8, requires States to establish a mandatory incident reporting system to collect actual or potential safety deficiency information. States shall also establish voluntary incident reporting that is nonpunitive and has protection for the sources of the information under Chapter 5 of Annex 19. Annex 19 further reinforces the obligation of incident investigation in the State Safety Program Element 1.3 - Accident and incident investigation:

The State has established an independent accident and incident investigation process, the sole objective of which is the prevention of accidents and incidents, and not the apportioning of blame or liability. Such investigations are in support of the management of safety in the State. In the operation of the SSP, the State maintains the independence of the accident and incident investigation organization from other State aviation organizations. 
This is also implemented by the service provider in the SMS Components related to Safety Risk Management, Safety Assurance and Safety Promotion.

State Safety Risk Management - Service provider's safety requirements for SMS

"The State has established the controls which govern how service providers will identify hazards and manage safety risks."

State Safety Assurance - Safety data collection, analysis and exchange

"The State has established mechanisms to ensure the capture and storage of data on hazards and safety risks at both an individual and aggregate

State level. The State has also established mechanisms to develop information from the stored data, and to actively exchange safety information with service providers and/or other States as appropriate."

State Safety Promotion - External training, communication and dissemination of safety information

The State provides education and promotes awareness of safety risks and two-way communication of safety-relevant information to support, among service providers, the development of an organizational culture that fosters an effective and efficient SMS.

A sample case study of the implementation of a safety investigation or review is provided in the appendix. The ICAO Safety Management Manual Document 9859 provides additional emphasis into a service provider's safety practices through the Eight Building Blocks identified in Section 3.7.1 (ICAO, 2013a). These are further reinforced in Appendix 1 of ICAO Annex 19. A service provider must be able to identify a deficiency before it can be managed. The periodic measurement of safety indicators will allow the development of trends and assist management in the allocation of resources. Safety performance measurements can be used to document both safety performance and efficiency performance through the need for fewer spare equipment or personnel due to fewer damaged equipment or personnel injuries (UK, 2006, September). Such continuous monitoring of normal operations for the identification of abnormalities or exceedences is an important step. Dissemination of lessons learned and best practices through the exchange of safety information enhances safety awareness, builds an open or positive safety culture and further supports the identification of safety issues. This is also referred to as a Just Safety Culture because management and employees have a clear understanding of acceptable and 
unacceptable behaviour and how unacceptable behaviour might result in sanctions under ICAO Annex 19, Appendix 2.

The Australian Civil Aviation Safety Agency (CASA) has provided guidance for the development of its SMS and the investigation of safety deficiencies by service providers in its CASA SMS Toolkit Booklet 4 Safety Assurance. This includes incident and occurrence investigation, documentation and dissemination.

CASA Regulation (CASR) Part 142.200 establishes the responsibilities of a service provider's safety manager and includes the obligation to implement a SMS including corrective, remedial and preventative action; regular discussion with the chief executive officer the effectiveness of the SMS; and establish a continuous improvement system.

CSAR Part 142.265 establishes that the SMS must be a systemic approach to managing safety by the identification of deficiencies related to training, operations and human factors. This approach will include the organizational structure, accountability, policies and procedures necessary to manage safety in a systemic way during normal and emergency operations. The SMS will monitor and measure safety performance and conduct internal safety investigations in order to ensure the sustainability of the organization through the management of change and provide for continuous improvement.

\section{Investigation Organization and Management}

A safety review or investigation should be organized and managed so that the investigators can efficiently perform their various tasks. It is essential that an investigation proceeds systematically through all aspects of the incident even when causes are apparent. This may include the use of specialized outside experts.

A more complicated incident investigation management system may have several functional areas with several investigation teams. Depending on the magnitude and circumstances of the incident, there may be from two to ten technical investigation areas that might include: Witnesses, Weather, Air Traffic Services, Aircraft Structures, Aircraft Systems, Powerplants, Maintenance Records, Survival Factors, Human Performance, Aircraft Performance, and Flight 
Recorders. Smaller incidents may be performed by a single investigator who may be supplemented by specialists.

It is essential that all personnel with access to the information developed by the safety investigation understand and comply with the process for distribution of the finding in order to minimize speculation or inaccurate information. A central point of approval of all reports and announcement must be established and all inquiries sent to that person. Any premature dissemination of factual information, speculation or rumors can prejudice the investigation and dilute the organization's confidence in the investigation outcomes.

\section{Laboratory Testing of Aircraft Systems and Components}

In some cases, examination or testing of specific components will be required. The outside testing laboratory should follow the same policies and procedures for tests and component examinations as used for the service provider's investigation. Often the component manufacturer's facilities have specialized equipment to assist in such analysis; however, close supervision by the service provider's investigators should be used to ensure there is no real or perceived conflict of interest. Each component will be tagged with its name, part number, serial number and the incident identifier. The component should be protected for shipment and include the conditions under which the component was operated and any suspected or specific faults to be tested so as to help focus the efforts of the testing. At a minimum a telephone communication should be made with the testing agency to establish a test plan and requested evaluations. Simply sending a component for testing with a note that it failed is insufficient guidance. After the testing is completed, the results will be reviewed and incorporated into the investigation report.

\section{Documentation of Incidents and Occurrences}

All incident and occurrence reports should contain as much information as necessary to capture all relative information for inclusion in trend analysis programs for continuous improvement and monitoring. The report may include both immediate and systemic findings. The identification of findings does not imply fault or blame and shall not be used for administrative or civil action. Where outside organizations are included in the findings, there should be coordinated with such organizations prior to distribution of the report. The safety team should also be mindful of the access of the report by regulators and other parties, and where possible de-identify those involved. This may assist in the promotion and solicitation of future critical safety information. 
The safety team, and those organizations involved in the investigation, might identify safety deficiencies prior to the delivery of the final report. Where appropriate, action to correct these identified deficiencies should be initiated as soon as possible. It is also essential that a tracking system be implemented to monitor the mitigation and ensure the corrective actions remain sufficient.

\section{Findings and Recommendations for Mitigation of Deficiencies}

The format of the safety investigation report will follow local guidance. The circumstances of the incident or occurrence will determine the size and scope of the report. Documents that are required to support the facts, analysis, conclusions, and recommendations are often included in appendices to the report. In consultation with the safety team members, the organization's accountable executive is responsible for evaluation of the evidence gathered and the drafting of the report. While the investigation should strive to achieve concurrence with all of the factual information, there may be alternative views in the report in order to fully cover all contingencies. The accountable executive may also be assisted by a separate safety expert in review of the safety investigation to ensure the recommendations are sufficient to mitigate the risk of future incidents or occurrences to an acceptable level.

The report may contain administrative actions, such as changes to standard operating procedures. There may also be lessons learned to be included in indoctrination and training evolutions to improve aviation safety culture of the organization. A standardized method must be established to incorporate the safety recommendations and corrective actions throughout the organization and to ensure that those not present and new staff are made aware of these new procedures. These processes are part of a change management program within the organization (UK, 2013). Should the mitigation procedures prove inadequate, the safety team should revisit the report and determine what additional steps must be taken. Supporting outside organizations should also be informed of these new conditions for their consideration.

\section{Summary}

Traditional aviation accident and incident investigation methodology provides an exceptional foundation for SMS investigations through a holistic approach to identify safety deficiencies. This leads to a more robust safety risk assessment for the mitigation of such deficiencies. The just-culture evaluation of incident and occurrence investigation further demonstrates to the organization the 
International Journal of Aviation, Aeronautics, and Aerospace, Vol. 1 [2014], Iss. 1, Art. 1

value of continual safety improvement to better support the efficiency and sustainability and of the organization. 


\section{References}

Australian Transport Safety Board (ATSB). (2003). Transport Safety Investigation Act 2003 Part 3 - Compulsory and Voluntary Reporting. Canberra, Australia: Author.

Civil Aviation Safety Agency. (2009a). Civil Aviation Advisory Publication (CAAP) - SMS-1. Safety Management System Requirements Regular Public Transport Operations. Canberra, Australia: Author.

Civil Aviation Safety Agency. (2009b). Civil Aviation Advisory Publication (CAAP) - SMS-2. Integration of Human Factors (HF) into Safety Management Systems (SMS). Canberra, Australia: Author.

Civil Aviation Safety Regulation (CASR) (2013). Part 142 - Integrated and multi crew pilot flight training and contracted recurrent training and checking. Canberra, Australia: Author.

European Union (EU.) (2010). The investigation and prevention of accidents and incidents in civil aviation. Investigation Regulation No 996/2010 Directive 94/56/EC.

Federal Aviation Administration (FAA) Advisory Circular (1976). AC 120-30A Reporting Requirements of Air Carriers, Commercial Operators, Travel Clubs, and Air Taxi Operators of Large and Small Aircraft. Washington, DC: Author.

FAA. (2010). Advisory Circular - AC 120-92A Introduction to SMSs for Aviation Service Providers. Washington, DC: Author.

FAA. (n.d.) Federal Aviation Regulation (FAR) Part 91 - General Operating and Flight Rules. Washington, DC: Author.

Gesell, L. E. \& Dempsey, P. S. (2011). Aviation and the Law, 5th ed. Chandler, AZ: Coast Aire Publications.

ICAO. (2003). Circular 298 - Training Guidelines for Aircraft Incident Investigators. Montreal, Canada: Author. 
ICAO. (2008). Circular 315 - Hazards at Aircraft Incident Sites. Montreal, Canada: Author.

ICAO. (2010). Annex 13 - Aircraft Accident and Incident Investigation. Montreal, Canada: Author.

ICAO. (2012). Manual of Aircraft Accident and Incident Investigation (Doc 9756). Montreal, Canada: Author.

ICAO. (2013a). Safety Management Manual ( $3^{\text {rd }}$ ed.) (Doc 9859). Montreal, Canada: Author.

ICAO. (2013b). Annex 19 - Safety Management. Montreal, Canada: Author.

Reason, J. (1990). Human Error. Cambridge, UK: Cambridge University Press.

United Kingdom, Aviation Accident Investigation Bureau (AAIB) (2013). Aircraft Accidents and Serious Incidents Guide for Airline Operations. Hampshire, UK: Author.

United Kingdom, Civil Aviation Authority (CAA). (2006). Civil Aviation Publication (CAP) 642 - Airside Safety Management. West Sussex, UK: Author.

United Kingdom, Civil Aviation Authority (CAA). (2013). Civil Aviation Publication (CAP) 1059 - Safety Management Systems: Guidance for small, non-complex organisations. West Sussex, UK: Author.

United States National Transportation Safety Board (NTSB) Part 830 Notification and reporting of aircraft accidents or incidents and overdue aircraft, and preservation of aircraft wreckage, mail, cargo and records. Washington, DC: Author. 


\section{Appendix}

\section{Case Study on employee nourishment:}

Hazard Identification - Through voluntary reports and observations from safety team members, it has been identified that some pilots and maintenance staff are exhibiting decreased performance near the end of their work schedule. In discussion with these groups, it was identified that the organization has not included the importance of adequate nourishment during induction and periodic human factors training as required by regulatory guidance.

\section{Safety Policy:}

The organization's SMS should provide a process for a proactive and integrated approach to Safety. This should include the active support of both employees and management. The Safety Policy should describe the structure and scope of the organization, resources, staff responsibilities and how safety decisions are managed throughout the organization. The current policy does not address nourishment.

\section{Safety Risk Management:}

Existing Mitigation - the organization has several vending machines that have candy and soft drinks along with a poster discussion proper hydration during the summer.

Current Level of Risk - because the organization is not near a restaurant, staff often bring food from home or rely on the snacks available. Also, because time for meals is not built into the work schedule, employees often simply eat at their desk or while walking to or from meetings. The severity of such lack of proper nourishment is moderate and the likelihood of the lack of nourishment is also moderate.

Risk Tolerability - the safety team has determined that the lack of proper nourishment and time to consume such nourishment has decreased the efficiency and safety of the organization to an Unacceptable Level requiring attention.

Further mitigation - the safety team has determined that an additional nourishment and training policy must be implemented. The vending company has been contacted to provide more nourishing options and a mobile catering company has been contacted to provide a hot meal during each shift.

Furthermore, the staff is encouraged to not eat meals at their desk and the lunch room has been expanded to provide free water, microwaves, and refrigerators.

Revised Level of Risk - following a test period, the safety team determined that the new policy, training and resources provided have achieved an acceptable level of safety. It was further noted that employee happiness and efficiency had increased. 


\section{Safety Assurance:}

The safety team monitors the induction and periodic training programs to ensure the continued emphasis on nourishment and hydration. The safety team also monitors regulatory guidance material for new programs or initiatives regarding nourishment.

\section{Safety Training and Promotion:}

The initial induction program now includes nourishment and hydration, with periodic promotion during changes in seasons and duty locations. The safety team also conducts an annual competition for employees and their families regarding nourishment, soliciting menus and posters to be used throughout the year to remind employees of the importance of proper nourishment and hydration. 